\section{Clinical photoacoustic imaging of cancer}

\author{
Keerthi S. Valluru, Juergen K. Willmann \\ Department of Radiology, Molecular Imaging Program at Stanford, Stanford University \\ School of Medicine, Stanford, CA, USA
}

Photoacoustic imaging is a hybrid technique that shines laser light on tissue and measures optically induced ultrasound signal. There is growing interest in the clinical community over this new technique and its possible clinical applications. One of the most prominent features of photoacoustic imaging is its ability to characterize tissue, leveraging differences in the optical absorption of underlying tissue components such as hemoglobin, lipids, melanin, collagen and water among many others. In this review, the state-of-the-art photoacoustic imaging techniques and some of the key outcomes pertaining to different cancer applications in the clinic are presented.

Keywords: Photoacoustic techniques; Neoplasms; Oncology; Spectroscopy, near-infrared

\section{Introduction}

The field of medical oncological imaging is experiencing a significant disruption with the advent of molecular imaging techniques that are aimed toward earlier detection as well as better characterization and monitoring of cancer [1-3]. Several types of medical imaging modalities are equipped with molecular imaging capabilities and are progressing towards mainstream cancer diagnostics $[4,5]$. They all share similar challenges in terms of regulatory, and reimbursement hurdles for clinical translation, and ultimately which imaging technique adds more value to the current standard of cancer care relies on its potential for global adoption. With increased affordability, availability, and portability of ultrasound machines, clinical ultrasound has now been deemed as a valuable diagnostic tool for resource-limited settings by the ministries of health in low and middle income countries, several nongovernmental organizations and the World Health Organization [6]. Therefore, technologies that can leverage ultrasound imaging platforms can potentially be adopted widely and more quickly compared to imaging technologies that are capital-intensive. Emerging techniques such as ultrasound molecular imaging [7-10], and ultrasound elastography [11-15] which are currently available as add-on features for ultrasound scanners have shown promise for early and more accurate detection of cancer compared to standard anatomical ultrasound.

Photoacoustic imaging is a hybrid technique that exposes the tissue with a laser and measures optically induced ultrasound signals $[16,17]$. Therefore, hardware unification of a clinical ultrasound scanner with an appropriate laser source will essentially result in a combined ultrasound and photoacoustic imaging device. In photoacoustic imaging, tissue absorbs pulsed near-infrared light and emanates ultrasound waves as it undergoes transient thermoelastic expansion. The photoacoustic conversion is proportional to the optical absorption and therefore individual tissue constituents that absorb light at different wavelengths can be selectively imaged with photoacoustic imaging

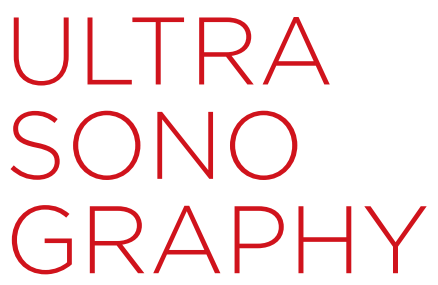

REVIEW ARTICLE

http://dx.doi.org/10.14366/usg. 16035 pISSN: 2288-5919 - eISSN: 2288-5943 Ultrasonography 2016;35:267-280

Received: August 10, 2016 Revised: August 30, 2016 Accepted: August 30, 2016

Correspondence to: Juergen K. Willmann, MD, Department of Radiology, Molecular Imaging Program at Stanford, Stanford University School of Medicine, 300 Pasteur Drive, Room H1307, Stanford, CA 94305-5621, USA

Tel. +1-650-725-1812

Fax. +1-650-723-1909

E-mail: willmann@stanford.edu

This is an Open Access article distributed under the terms of the Creative Commons Attribution NonCommercial License (http://creativecommons.org/ licenses/by-nc/3.0/) which permits unrestricted noncommercial use, distribution, and reproduction in any medium, provided the original work is properly cited.

Copyright (C) 2016 Korean Society of Ultrasound in Medicine (KSUM)

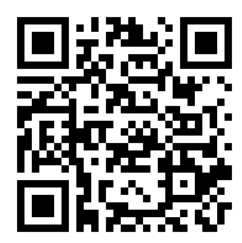

How to cite this article:

Valluru KS, Willmann JK. Clinical photoacoustic imaging of cancer. Ultrasonography. 2016 Oct;35(4):267-280. 
(spectroscopy). For example, with angiogenesis being one of the hallmarks of cancer [18], spectroscopic photoacoustic imaging can reveal tissue physiology and help detect neoangiogenesis based on the differences in the absorption of hemoglobin in cancerous tissue compared to the surrounding normal tissue [19]. Moreover, the discovery of cancer biomarkers and molecular targeted contrast agents [20] is streamlining the efforts to detect cancer earlier and more accurately. By using molecularly targeted contrast agents with dyes or agents with known optical absorption properties, molecular photoacoustic imaging can be performed. These physiological and molecular capabilities of photoacoustic imaging have drawn a considerable interest in the medical imaging community resulting in many publications and patents.

In this review, the current status of clinically translatable stateof-the-art photoacoustic imaging techniques and some of the key outcomes pertaining to different cancer applications along with challenges and possible solutions are discussed.

\section{Basics of Photoacoustic Imaging}

The conversion of light to sound is called photoacoustic effect and has been first introduced by Alexander Graham Bell in 1880 [21]. When a tissue is exposed to high energy, short duration (nanoseconds) pulses of electromagnetic radiation such as laser light, it undergoes optical absorption which in turn causes localized heating and rapid thermoelastic expansion producing transient broadband ultrasound waves (Fig. 1). Since these ultrasound (-acoustic) waves are a result of tissue optical (-photo) absorption, they are generally referred to as photoacoustic waves and the technique of reconstructing the images from the signals corresponding to these photoacoustic waves is called photoacoustic (or optoacoustic) imaging [22]. Because photoacoustic imaging relies on light propagation only one-way and the detection is based on ultrasound generation, deeper tissue penetration is possible compared to traditional optical imaging techniques. Ultrasound scattering is two to three orders of magnitude weaker than optical scattering in tissue [17] allowing deeper tissue imaging and, therefore, provides images with high spatial resolution representing tissue optical absorption. Moreover, as the image formation is based on tissue absorption rather than scattering, the photoacoustic images are also free of speckle which is commonly seen on ultrasound [23]. With other obvious advantages like nonionizing laser radiation, affordability and ease of integration into clinical ultrasound scanners, and the ability to provide physiological information leveraging endogenous tissue contrast, photoacoustic imaging can potentially transform the clinical imaging landscape by complementing existing techniques for improved cancer imaging.
A typical biomedical photoacoustic imaging system consists of a pulsed tunable near infrared (NIR) wavelength laser with pulse width ( $<10$ nanoseconds), fluence $\left(20-100 \mathrm{~mJ} / \mathrm{cm}^{2}\right.$ at $700-1,100$ $\mathrm{nm}$ ) and pulse repetition frequency (PRF; $10 \mathrm{~Hz}-50 \mathrm{~Hz}$ ) to achieve high quality real-time images of tissue. The NIR wavelength range is important for medical imaging because the optical absorption by tissue is minimal in this spectrum allowing the light to penetrate deeper into the tissue [24]. Shorter pulse widths ensure that the stress and thermal confinement conditions are met warranting the photoacoustic effect while higher fluence (energy delivered per unit area) yields better imaging depth and increased photoacoustic signal generation in tissue. There is no hard limit for the PRF except that the higher rates account for faster imaging time, and thus are better for clinical applicability.

Typically, the photoacoustic waves that are generated from an optical absorption event propagate away from the source as

\section{Ultrasound}

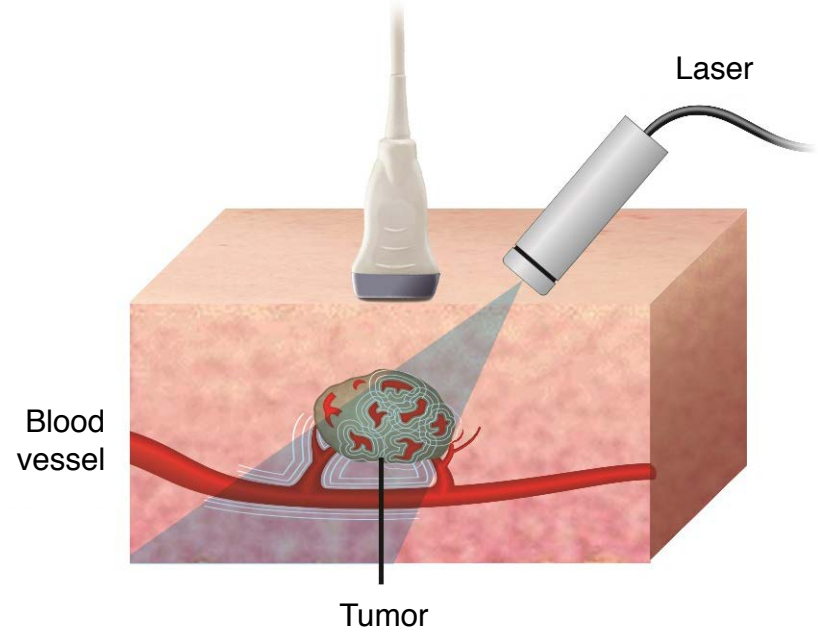

Fig. 1. Principle of photoacoustic effect and imaging. When a tissue is exposed to pulsed near infrared laser, the tissue constituents such as hemoglobin, lipid, water, collagen, etc. absorb light and undergo thermoelastic expansion, thereby emanating ultrasound signals (photoacoustic effect). An ultrasound transducer can thus detect these laser-induced ultrasound signals (photoacoustic signals) and facilitate photoacoustic (or optoacoustic) imaging. Each of these bio-absorbers can be targeted by irradiating tissue at its corresponding dominant absorption wavelength. Hence, by using a tunable laser operating at the relevant wavelengths of interest, one can obtain multiple photoacoustic images that can be spectrally resolved to allow assessment of the tissue composition based on endogenous contrast. For example, by assessing blood oxygenation in the tissue, contrast obtained from photoacoustic imaging can potentially be helpful in detection, characterization, and monitoring of cancer. 
spherical transients and can be detected using an ultrasound transducer placed at a distance (over the surface of the skin). Consequently, the most common and affordable approach to build a photoacoustic imaging system is to modify a clinical ultrasound scanner by integrating it with a pulsed NIR laser with an appropriate light delivery mechanism to stimulate the tissue and detect the photoacoustic waves simultaneously [25]. The key is to synchronize the transducer acquisition time with laser firing to ensure accurate registration and spatial localization of the absorption event. Since a clinical ultrasound scanner is already equipped for ultrasound imaging, this approach has the advantage of providing co-registered ultrasound B-mode and photoacoustic images simultaneously. A key limitation, however, is that a traditional ultrasound transducer array can only detect a portion of the spherical photoacoustic transients posing a tradeoff in image contrast (Fig. 2). Alternatively, an approach called photoacoustic computed tomography [26] can result in the acquisition of a larger portion of the signal either by mechanically scanning a focused ultrasound transducer over the tissue or using transducers arranged in a curved array around the tissue coupled with an image reconstruction scheme where photoacoustic signals from all the transducer elements are filtered, back-projected and added to form a final image similar to $X$-ray computed tomography. This allows greater control over photoacoustic data acquisition providing time-dependent radiofrequency (containing amplitude, phase, and frequency) data that can be used for image quantification. However, photoacoustic tomography often requires a dedicated photoacoustic imaging system due to the transducer arrangement necessitating custom hardware and software for image reconstruction.

Another decisive step from the application standpoint is the selection of imaging wavelengths. Optical absorption of tissue constituents is discrete at NIR wavelengths ranging from 700 to $1,100 \mathrm{~nm}$ where light penetration of up to several centimeters is possible $[27,28]$. Spectroscopic photoacoustic imaging employs multiple wavelengths of laser light to determine the relative concentrations of specific chromophores in tissue such as hemoglobin, lipid, water, melanin, and collagen among others $[19,29-33]$. This ability to spectrally resolve relative amounts of deoxygenated and oxygenated hemoglobin to determine hemoglobin concentration and oxygen saturation for example [34], has been the basis for several studies aiming to characterize cancerous tissue which is often hypoxic compared to noncancerous tissue $[35,36]$. Beyond imaging endogenous absorbers, spectroscopic photoacoustic imaging has been investigated to evaluate how certain exogenous contrast agents such as dyes [37-41], nanoparticles [42,43], carbon nanotubes [44], nanodroplets [45,46], liposomes, and polymer particles [39] are biologically processed in vivo and contribute to the overall improvement of photoacoustic image contrast. These exogenous contrast agents when conjugated or encapsulated with suitable antibodies or peptides and administered intravenously, can bind to cancer-specific biomarkers that do not naturally absorb light facilitating photoacoustic molecular imaging [47].

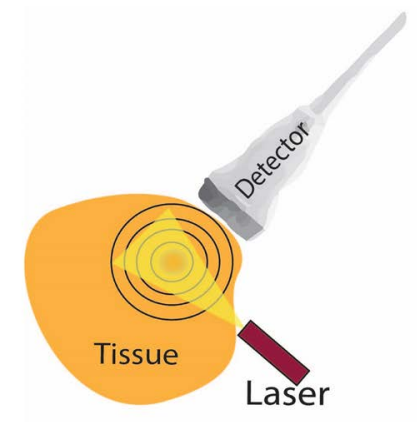

A

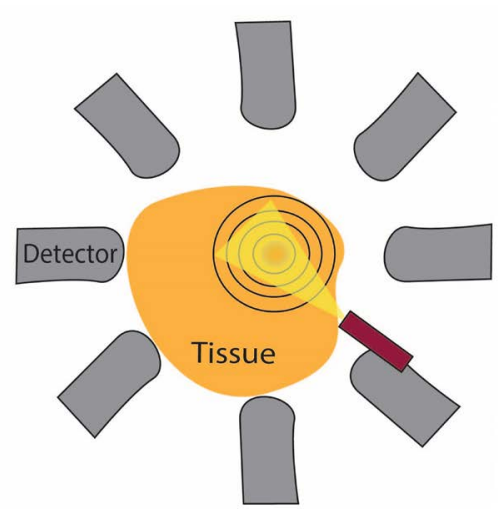

B

Fig. 2. Photoacoustic imaging configurations.

Laser source and the detectors may be on the same side or at an angle to each other. However, an overlap must exist between the laser beam and the detector's directivity pattern to ensure that laser-induced ultrasound signals are detected and visualized. A. Photoacoustic imaging performed with a conventional ultrasound transducer where only a portion of the spherical wavefront (black rings) originated from the target (red absorber at the center of the rings) is seen by the transducer. B. Photoacoustic tomography approach showing X-ray computed tomography-like reconstruction where a single detector can be rotated around the target or an array of multiple stationary detector elements around the target can be employed. The signal arriving at each detector is filtered back-projected along circular arcs in the spatial domain, and then all the back-projections are added together to obtain the final photoacoustic image which represents the spatial distribution of optical absorption within the target. 


\section{Photoacoustic Imaging of Cancer: Clinical Applications}

Photoacoustic imaging has traditionally been implemented with systems that can be broadly categorized into either stand-alone photoacoustic imaging systems with linear/phased array transducers, or photoacoustic computed tomography systems which commonly employ curved array transducers and custom data acquisition units. Many of these systems are being transitioned from their initial prototype design to assume a more practical form factor of clinical ultrasound scanners with handheld probes. A review of selected studies involving cancer patients is provided to give a glimpse of photoacoustic imaging technologies/applications that are clinically translatable, and the readers are encouraged to refer to recent reviews for more information on other applications [16,48-52].

\section{Stand-Alone Photoacoustic Imaging Systems}

Photoacoustic imaging has been explored by several investigators for breast imaging as a potential alternative to $X$-ray mammography which is the standard-of-care screening technique in American women of age 40 years or older [53]. As such, these mammographyalternative devices were often developed under the presumption that the healthcare system will embrace the shift from X-ray mammography to modalities that are comparable in cost, but add significant value to the patient. These photoacoustic mammography systems typically have a patient examination table with an aperture to suspend the patient's breast when lying in prone position. And the laser and detector are arranged underneath the exam table with the breast mildly compressed. One such systems is the photoacoustic mammoscope, a prototype clinical system developed at the University of Twente (Enschede, Netherlands) [54]. This system employs a two-dimensional circular ultrasound detector array (80 $\mathrm{mm}$ diameter, 590 elements, 1-MHz center frequency) on one side of the compressed breast with laser exposure $\left(1,064 \mathrm{~nm}, 10 \mathrm{~mJ} / \mathrm{cm}^{2}\right)$ from the other side to facilitate three-dimensional (3D) imaging. Results from a recent clinical study (Fig. 3) with 43 patients (31 malignant, 2 fibroadenomas, 1 chronic inflammation, 5 cysts, and 2 invalid measurements) reported the visualization of tumors with high contrast in 30/31 breast cancer patients (Breast Imaging Reporting and Data System [BI-RADS] density: low [1 or 2] in 23 patients and high [3 or 4] in 8 patients) concluding that the photoacoustic image contrast was independent of the mammographically estimated breast density unlike X-ray mammography [55]. The average scan time with photoacoustic mammoscope was 10 minutes for a field of view of $90 \times 80 \mathrm{~mm}^{2}$. Based on a subjective survey of 30 patients involved in the study, 25 patients indicated their preference to photoacoustic mammography over $\mathrm{X}$-ray mammography in terms of comfort. Upon a formal health technology assessment of photoacoustic mammoscope [56], photoacoustic imaging was further determined as the most promising alternative to the combined use of X-ray mammography and ultrasonography for early stage breast cancer diagnosis.

Another stand-alone photoacoustic imaging system (Canon Inc., Tokyo, Japan) introduced by Kitai et al. [57] is similar to the photoacoustic mammoscope in principle where the patient lies in prone position on the examination table with the breast suspended through a rectangular aperture $(17 \mathrm{~cm} \times 18 \mathrm{~cm})$ and mildly compressed between two plates craniocaudally underneath the table. Laser delivery (4 wavelengths: 756 nm, $797 \mathrm{~nm}, 825 \mathrm{~nm}$, and $1,064 \mathrm{~nm}$ ) onto the breast is achieved through both plates (dualillumination) while the rectangular detector array $(15 \times 23$ elements, $1 \mathrm{MHz}$ ) is coupled to the caudal plate to facilitate $30 \mathrm{~mm} \times 46 \mathrm{~mm}$ scans in 45 seconds. A total of 42 lesion-bearing breasts from 40 patients were imaged with this system who also underwent mammography, magnetic resonance imaging, ultrasound, and eventually breast surgery [58]. Histologically, 39/42 lesions were found to be malignant with 33 of them classified as invasive breast cancers while $3 / 42$ were benign. Out of the 39 malignant lesions, 29 lesions (74.4\%) were identified to be microvessel rich using photoacoustic imaging. Mean oxygen saturation was also calculated in breast tumors for each patient by processing multi-wavelength images, and all the measured lesions showed lower oxygen saturation levels compared to surrounding vasculature $(P<0.001)$ within the target breast, as well as the normal contralateral breast $(\mathrm{P}=0.001)$.

Kim et al. [59] explored the use of photoacoustic imaging to detect breast microcalcifications as an alternative to mammography, avoiding radiation exposure. Twenty-one biopsy cores from 16 patients: 11 cores with microcalcifications from 11 patients suspected to have malignancy by mammography, and 10 cores without microcalcifications from five patients with biopsy-confirmed fibroadenoma, were evaluated using their custom-designed photoacoustic imaging system. The biopsy core specimens were soaked in saline solution for about 6 hours to wash off any blood and imaged with their system (dual wavelength laser: $700 \mathrm{~nm}$ and $800 \mathrm{~nm}, 7$ nanoseconds, $10 \mathrm{~Hz}$; detector: 7-MHz linear array scanned along elevation direction to acquire 3D data) in order to spectrally enhance the signal obtained from microcalcifications. Microcalcifications were hypothesized to show a signal with maximum amplitude at $700 \mathrm{~nm}$, and a decreased or diminished amplitude at $800 \mathrm{~nm}$ while this difference is expected to be less pronounced in nonmicrocalcifications. Based on the ratio between signals at $700 \mathrm{~nm}$ to signals at $800 \mathrm{~nm}, 10 / 11$ specimens containing microcalcifications (median ratio, 2.46), and 8/10 specimens without 

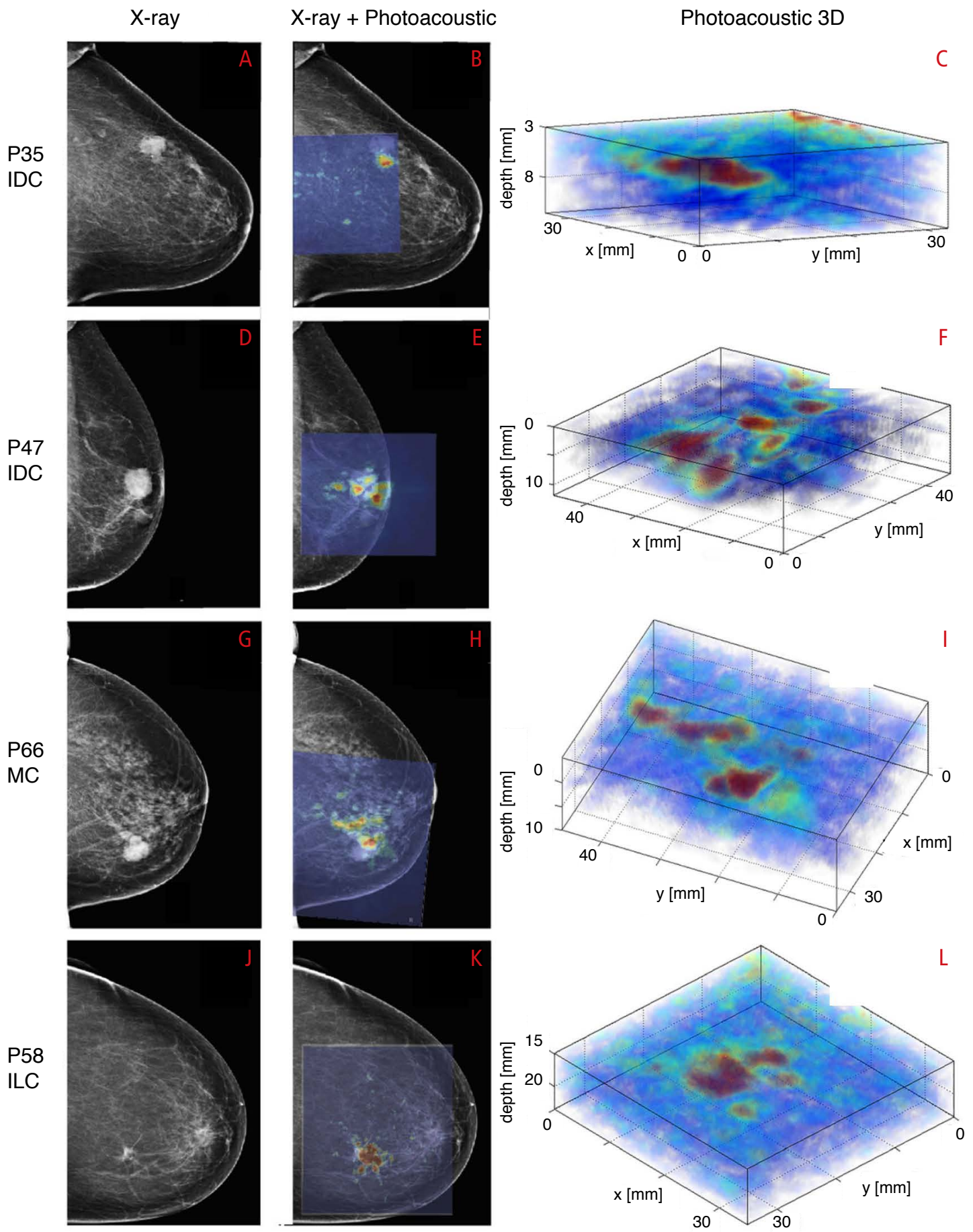

Fig. 3. Breast imaging with photoacoustic mammoscope.

Photoacoustic images were overlaid on X-ray mammograms to show lesions detected on both modalities. Reconstructed 3D photoacoustic volume encompassing each lesion of interest is also shown here. Infiltrating ductal carcinoma (IDC) lesion was seen on X-ray and photoacoustic imaging in a 79-year-old patient $(A-C)$ and a 69-year-old patient $(D-F)$, respectively. Mucinous carcinoma (MC) was detected in an 83-year-old patient (G-I) while infiltrating lobular carcinoma (ILC) was seen in a 65-year-old patient $(\mathrm{J}-\mathrm{L})$. The lesions were co-localized on photoacoustic images with respect to X-ray mammograms and were visualized at depths of more than $20 \mathrm{~mm}$ with good contrast on photoacoustic images. PXX indicates patient-identifier in the study. Reproduced from Heijblom M et al. Eur Radiol 2016, http:// dx.doi. org/10.1007/s00330-016-4240-7 [55], according to the Creative Commons license. 
microcalcifications (median ratio, 1.10) were correctly identified with their system $(P=0.006)$, when evaluated against specimen mammography results.

The application of photoacoustic imaging to characterize skin lesions was investigated by Favazza et al. [60] using a standalone photoacoustic microscopy system which consisted of a dye laser (570 nm and $700 \mathrm{~nm}, 500 \mathrm{~Hz}$ ) and a 50-MHz single element ultrasound transducer (V214-BB-RM, Panametrics Inc., Waltham, MA, USA) connected to a motorized translation stage. When the system was utilized to scan the forearm of a human volunteer, it was able to differentiate a benign melanocytic nevus (mole) from the surrounding blood vessels. To acquire a three-dimensional volumetric image of a $6 \mathrm{~mm} \times 4 \mathrm{~mm}$ area with nevus, it took 5-10 minutes as the system scanned at $20 \mu \mathrm{m}$ increments in horizontal and vertical directions. From the photoacoustic images, the dimensions of nevus were measured as 262-270 $\mu \mathrm{m}$ thick, 2.64-2.66 mm wide, and located 135-140 $\mu \mathrm{m}$ deep below the skin surface comparable to the histological measurements demonstrating the potential of photoacoustic imaging in detecting skin lesions.

Urological and endocrinological applications of photoacoustic imaging were explored by Dogra et al. [61] using a stand-alone photoacoustic imaging system that can achieve real-time focusing using an acoustic lens instead of traditional electronic focusing methods. The system was reported to employ a tunable pulsed laser (760 nm, 850 nm, $930 \mathrm{~nm}$, and $970 \mathrm{~nm}$ ) for spectroscopic imaging and a linear ultrasound array (32 elements, $5 \mathrm{MHz}$ ) coupled with a data acquisition unit which was capable of 3D volumetric image acquisition. The scan time was estimated as 5 minutes to image an area of $45 \mathrm{~mm} \times 45 \mathrm{~mm}$. With this system, the research group performed two independent ex vivo studies involving prostate and thyroid cancer patients. In the prostate study, 42 grossly sectioned prostate specimens were acquired immediately after prostatectomy from 30 patients with biopsy-confirmed prostate cancer [62]. Upon spectroscopic decomposition, photoacoustic images of normal, benign prostatic hyperplasia (BPH), and malignant specimens were reconstructed indicating the presence of deoxyhemoglobin, oxyhemoglobin, lipid, and water. When correlated with histopathology, the results revealed that the system was able to identify 13/16 malignant and 25/26 nonmalignant (BPH and normal combined) prostate samples based on the deoxyhemoglobin signature. Furthermore, significant differences in mean intensity values of deoxyhemoglobin and lipid were reported between malignant and normal prostate. Similarly, substantial differences in mean intensity of deoxyhemolgobin between malignant prostate and BPH was seen while no statistically significant difference in any of the constituents was reported between $\mathrm{BPH}$ and normal prostate tissue. Overall, when BPH and normal prostate tissue were considered together as nonmalignant prostate, a statistically significant difference in mean intensity of deoxyhemoglobin was found between malignant and nonmalignant prostate tissue. In the thyroid study [63], spectroscopic photoacoustic imaging was performed immediately following resection of 88 thyroid lesions (13 malignant and 75 benign) harvested from 50 patients who underwent total or partial thyroidectomy. When a similar analysis to prostate study was performed on this dataset, an increase in mean photoacoustic signal intensity of de-oxyhemoglobin was reported in malignant thyroid tissue compared to benign thyroid tissue. The sensitivity and specificity in differentiating malignant from nonmalignant thyroid tissue were reported as $69.2 \%$ and $96.9 \%$, respectively.

Likewise, Aguirre et al. [64] have investigated gynecological applications of photoacoustic imaging with their customdeveloped photoacoustic imaging system. This system incorporated a pulsed tunable laser $(740 \mathrm{~nm}, 12$ nanoseconds, $15 \mathrm{~Hz}$ ) and a 1.75-dimensional transducer array ( $5 \mathrm{MHz}$ ) and was used to perform an ex vivo study on 33 human ovaries extracted from patients who underwent oophorectomy. Results of their study indicated that it was possible to differentiate normal postmenopausal versus malignant postmenopausal ovaries with a sensitivity and specificity of $83 \%$ and $83 \%$, respectively. For clinical use, an upgraded system was later introduced by the investigators $[65,66]$ employing a laser (750 nm, 20 nanoseconds, $15 \mathrm{~Hz}$ ) and a modified commercial transvaginal ultrasound probe $(6 \mathrm{MHz})$ to accommodate fiber optic cables that can deliver laser onto the ovaries through the probe. However, an in vivo study involving ovarian cancer patients is not yet reported to date.

Cancer of the cervix is another application that has been investigated by Peng et al. [67] who employed a photoacoustic imaging system (532 nm laser delivered through optical fiber with spot size $1 \mathrm{~mm}$ ) with a $10-\mathrm{MHz}$ focused transducer for signal detection. An in vitro study was carried out on frozen cervical biopsy samples (3-6 mm in size) collected from 30 patients who underwent cervical colposcopical screening. Each frozen sample was divided into two halves, each of which was evaluated by photoacoustic imaging and histopathology (Fig. 4). Each frozen sample consisting of normal tissue and lesion was embedded in a cylindrical agar phantom at a depth of $5 \mathrm{~mm}$ from surface to mimic optical properties of the human cervix and was imaged with the system. Histological staging confirmed that there were $1 / 30$ cervical intraepithelial neoplasia (CIN) 2, 6/30 CIN3, 12/30 cervical cancer (CC) 1, 10/30 CC2, and 1/30 CC3 samples (CIN, CC). Upon correlation with histopathology, photoacoustic images indicated an increased mean optical absorption in cervical lesions compared to normal cervical tissue samples. Also, the mean optical absorption 
was observed to increase monotonically with the severity of lesions yielding a correlation of $94 \%$ between staging of CC and the mean optical absorption.

\section{Photoacoustic Computed Tomography Systems}

Using a photoacoustic computed tomography approach, Ermilov et al. [68] developed a system called Laser-based Optoacoustic Imaging System (LOIS-64) which employs an annular array of detectors (64 elements) arranged in a hemispherical cup in which the patient's breast is suspended. The system utilizes a NIR laser (755 $\mathrm{nm}, 10 \mathrm{~mJ} / \mathrm{cm}^{2}$ ), and a custom image acquisition and processing system to enable tomography. Results from a clinical study involving

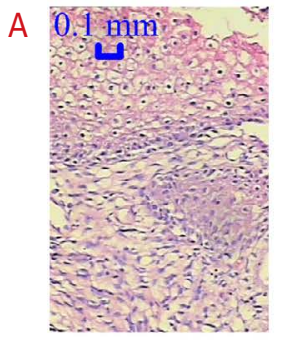

B
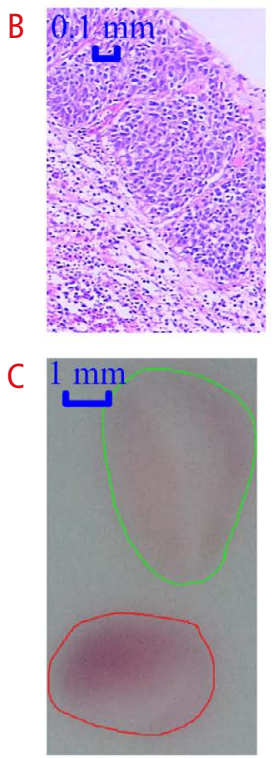

D

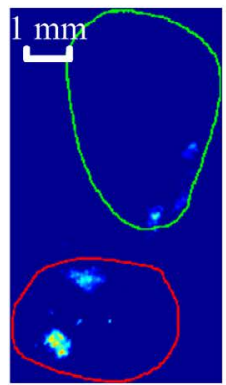

CIN 2
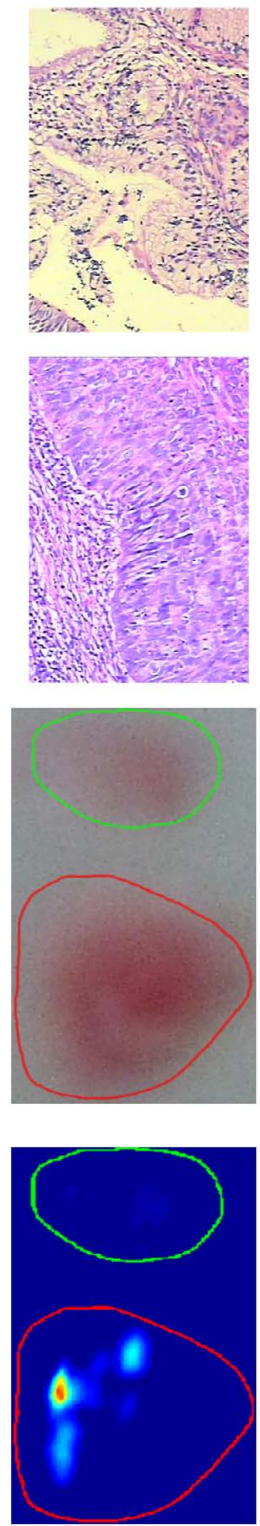

CIN 3
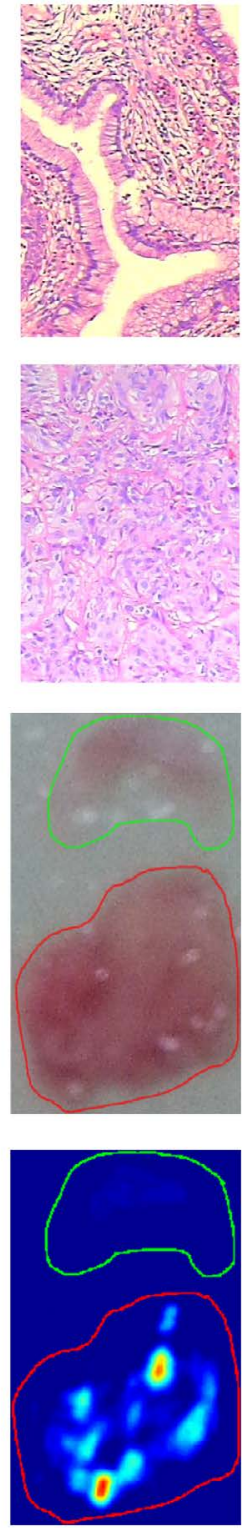

CC 1
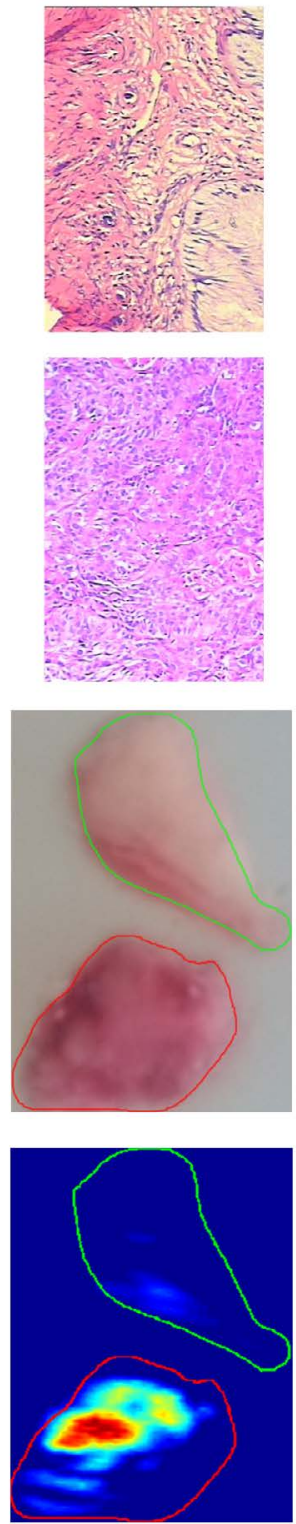

CC 2
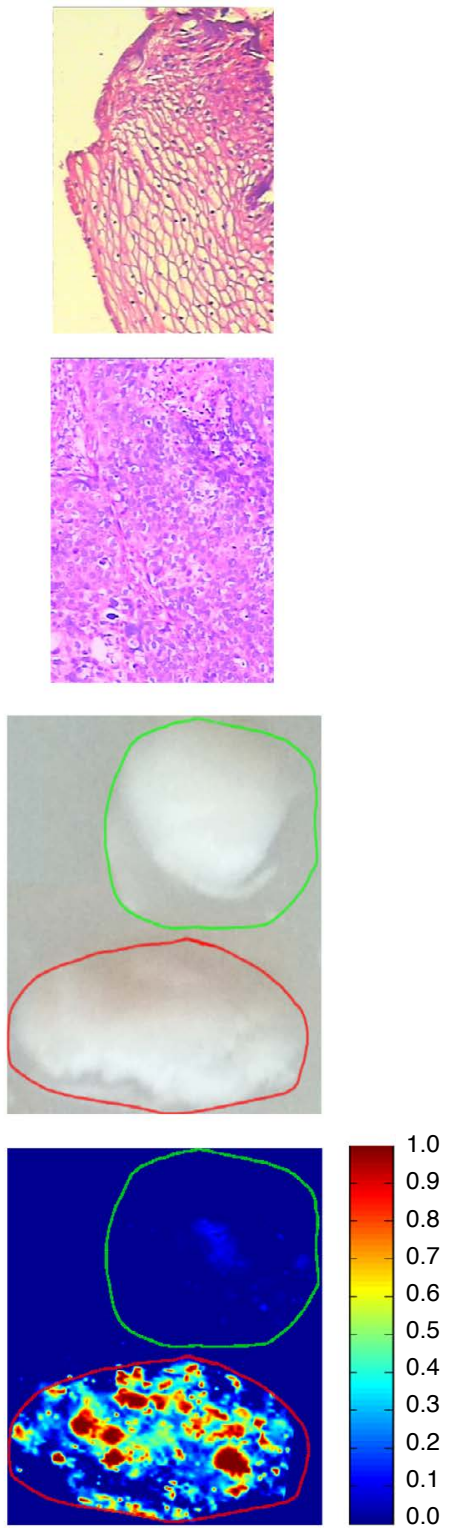

$\mathrm{CC} 3$

Fig. 4. Photoacoustic imaging of human cervix ex vivo.

Each frozen cervical tissue sample consisting of normal tissue and lesion was embedded in a cylindrical agar phantom. Histopathology images showing normal cervical tissue (A) and cervical lesion (B). Photographs of gross tissue specimens embedded in agar showing normal tissue on the top and lesion on the bottom (C). Corresponding photoacoustic images (D) showing signal intensity proportional to the lesion staging. Normal tissue is marked in green and cervical lesion is marked in red. The scale bar for the histological images represents $0.1 \mathrm{~mm}$, and that for the photographs and photoacoustic images is $1 \mathrm{~mm}$. CIN, cervical intraepithelial neoplasia; CC, cervical cancer. Reprinted from Peng K et al. Biomed Opt Express 2015;6:135-143 [67], with permission of Optical Society of America. 
27 patients suggested that $18 / 20$ biopsy-confirmed malignant lesions previously suspected on X-ray mammography and ultrasound were visualized by LOIS-64 [68].

A similar system was developed by Kruger et al. [69] which consists of a Food and Drug Administration (FDA)-approved $756 \mathrm{~nm}$ laser and an examination table with circular aperture comprising of a hemispherical array of spirally arranged ultrasound detectors (512 circular elements, $2 \mathrm{MHz}$ ). The feasibility of clinical breast imaging with this system utilizing photoacoustic computed tomography was reported using four healthy patients. The scan time with this system was between 12 seconds and 3.2 minutes depending on the active spiral size of the detector ranging from 24-mm to $96-\mathrm{mm}$ radius.

Besides breast imaging application, an ex vivo study of lymph nodes collected from patients with cutaneous melanoma who also underwent lymphadenectomy was reported to stage melanoma [70]. The photoacoustic computed tomography system employed a topdown laser delivery scheme $(720$ nm, 760 nm, 800 nm, 850 nm, and $12 \mathrm{~mJ} / \mathrm{cm}^{2}$ ) with the sample in between, and the imaging was performed by rotating a curvilinear ultrasound array (32 elements, $6.25 \mathrm{MHz}$ ) around the sample. The acquisition time with this system was reported as 4 minutes per wavelength during which 18 projections of each lymph node were acquired to reconstruct a final image. Results from the ex vivo lymph nodes resected from one patient suggested that their system was able to detect strong photoacoustic signal intensity throughout the lymph node which was confirmed as melanoma through histopathology evaluation.

A similar but larger study on 148 sentinel lymph nodes (SLNs) excised from 65 patients was performed using multispectral optoacoustic tomography (MSOT) inVision 128 system (iThera Medical GmbH, Munich, Germany), a commercially available preclinical system to detect melanoma metastasis [71]. 3D multispectral imaging was performed at 10 wavelengths between 700 and $880 \mathrm{~nm}$ (PRF $10 \mathrm{~Hz}$, averaged over 25 laser pulses) using an arc-shaped $\left(270^{\circ}\right)$ transducer array ( $5 \mathrm{MHz}, 128$ elements). Out of 148 SLNs, 77 SLNs showed a photoacoustic signal corresponding to melanin with 34 of them confirmed as metastatic by histopathology (stained for Melan A). The other 71 SLNs revealed no photoacoustic melanin signal and were deemed to be nonmetastatic histologically, indicating a system sensitivity of $100 \%$ and a specificity of $62 \%$ in detecting SLNs.

\section{Photoacoustic Imaging Systems Resembling Clinical Ultrasound Scanners}

Imagio (Seno Medical Instruments, Inc., San Antonio, TX, USA) was the first photoacoustic imaging system to receive a regulatory clearance (CE mark) and is commercially available in Europe. Imagio is a dual-laser system $(1,064 \mathrm{~nm}$ and $755 \mathrm{~nm})$ intended for breast cancer diagnosis and is equipped with a handheld commercial linear ultrasound array (128 elements, $5 \mathrm{MHz}$ ) to allow real-time data acquisition [72]. Currently, this system is being validated through a large, multi-center prospective clinical trial in the United States with an estimated enrollment of 2,000 breast cancer patients. An interim report of this ongoing trial reported that 66 patients (37 cancers and 29 benign lesions proven histologically) were imaged successfully with a sensitivity and specificity of $100 \%$ and $72 \%$ $79 \%$, respectively $[73,74]$.

A related application with the goal to noninvasively detect SLNS that can help determine the stage and extent of metastasis of breast cancer was explored on a clinical ultrasound scanner (iU22, Phillips Healthcare, Andover, MA, USA) modified for photoacoustic imaging by incorporating a laser $\left(667 \mathrm{~nm}, 10 \mathrm{~mJ} / \mathrm{cm}^{2}\right)$ and a handheld linear array (4 to $8 \mathrm{MHz}$ ) enabling co-registered ultrasound and photoacoustic imaging $[75,76]$. Sixteen patients with pathologicallyconfirmed breast cancer scheduled for axillary lymph node dissection were imaged with this system to achieve SLN visualization based on increased contrast from methylene blue injection. The goal of this study was to detect SLNs using an alternative noninvasive, nonionizing approach to the traditional SLN biopsy, an invasive surgical procedure that also involves radioactive colloid injection. In all patients, methylene blue $(5 \mathrm{~mL}, 2 \mathrm{mg} / \mathrm{mL}$ ) was injected subcutaneously near the areola in the same breast quadrant as the primary tumor and allowed to drain for 5 minutes followed by combined photoacoustic and ultrasound imaging. Thereafter, a small titanium clip was deployed in 13 patients under photoacoustic image-guidance to mark suspected SLNs. After the imaging session, patients underwent conventional SLN biopsy using both methylene blue and radioactive colloid injections. The SLNs were later removed surgically and imaged with specimen radiography followed by histopathological examination (Fig. 5). The presence of titanium clip within the SLN specimen was confirmed in 6/13 patients suggesting the feasibility of photoacoustic imaging in detecting SLNs that can be sampled using minimally invasive fine needle aspiration biopsy to determine breast cancer metastasis.

In a similar SLN study aimed at detection of melanomatic metastasis, 20 patients underwent radioactive lymphoscintigraphy and single-photon emission computed tomography/computed tomography (SPECT/CT) before photoacoustic imaging [71]. For this study (Fig. 6), the authors employed a handheld photoacoustic imaging system with two detector configurations [77]: (1) cylindrically focused 256-element, 4-MHz, arc-shaped $\left(135^{\circ}\right)$ detector array for $25 \times 25 \mathrm{~mm}^{2}$ planar acquisition and (2) semispherical 384-element, $2.5-\mathrm{MHz}$ detector for $20 \times 20 \times 25 \mathrm{~mm}^{3}$ volumetric acquisition. Multispectral imaging was performed at five wavelengths $(700,730,760,800$, and $850 \mathrm{~nm})$ with one laser 
pulse per wavelength (PRF $20 \mathrm{~Hz}$ ). This system is currently available for exploratory clinical use as MSOT Acuity (iThera Medical $\mathrm{GmbH}$ ) with optional integrated photoacoustic/ultrasound detectors $[78,79]$ and is yet to receive its CE Mark and FDA clearance for commercial adoption. By using Indocynanine Green as localizing contrast agent, photoacoustic imaging was able to visualize 41 SLNs (100\%) in 20 patients previously identified with lymphoscintigraphy and SPECT/CT. In addition to SLN detection, the ability of photoacoustic imaging to noninvasively detect melanoma metastases was examined based on melanin content within the node in the same study [71]. Eighteen of
41 SLNs (43.9\%) were considered nonmetastatic by photoacoustic imaging and histology, 19/41 (46.4\%) showed melanin signal on photoacoustic imaging but stained negative for Melan A (false-positive for metastasis attributed to pigmentation, nevi, melanophages, or hemorrhage), and 4/41 (9.7\%) were true-positive as confirmed by histopathology. While radiation-free SLN detection was achieved successfully, the high false-positive rate for metastasis detection emphasizes the need to excise SLNs and perform histopathology assessment for accurate cancer diagnosis until an alternative melanoma-specific contrast agent is available. With no
0

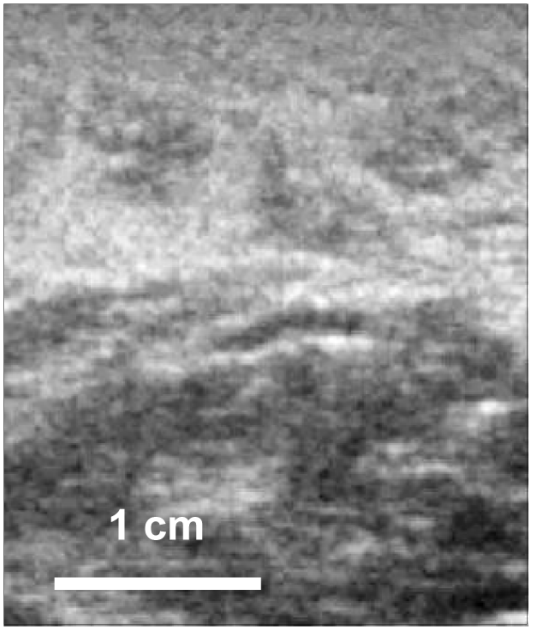

A

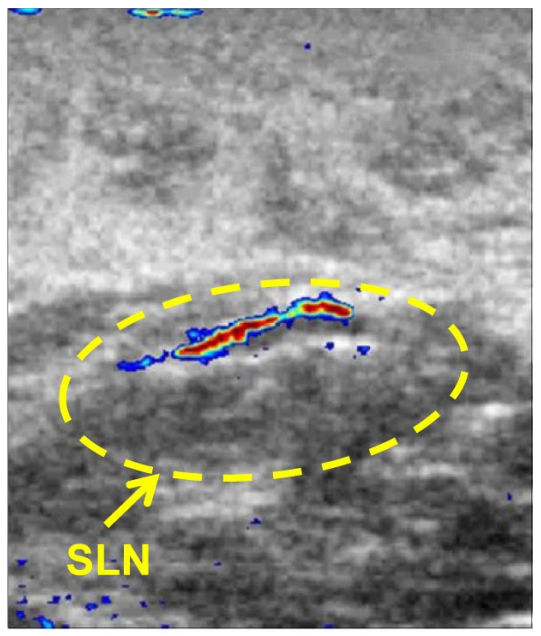

C

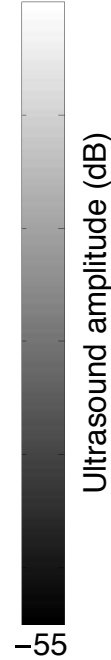

1

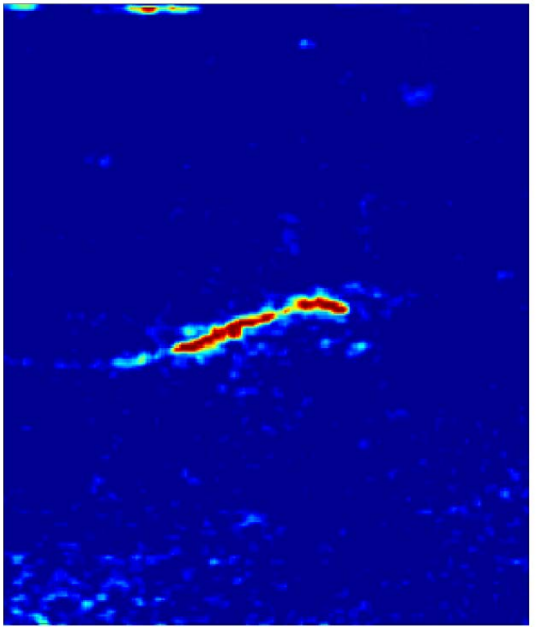

B
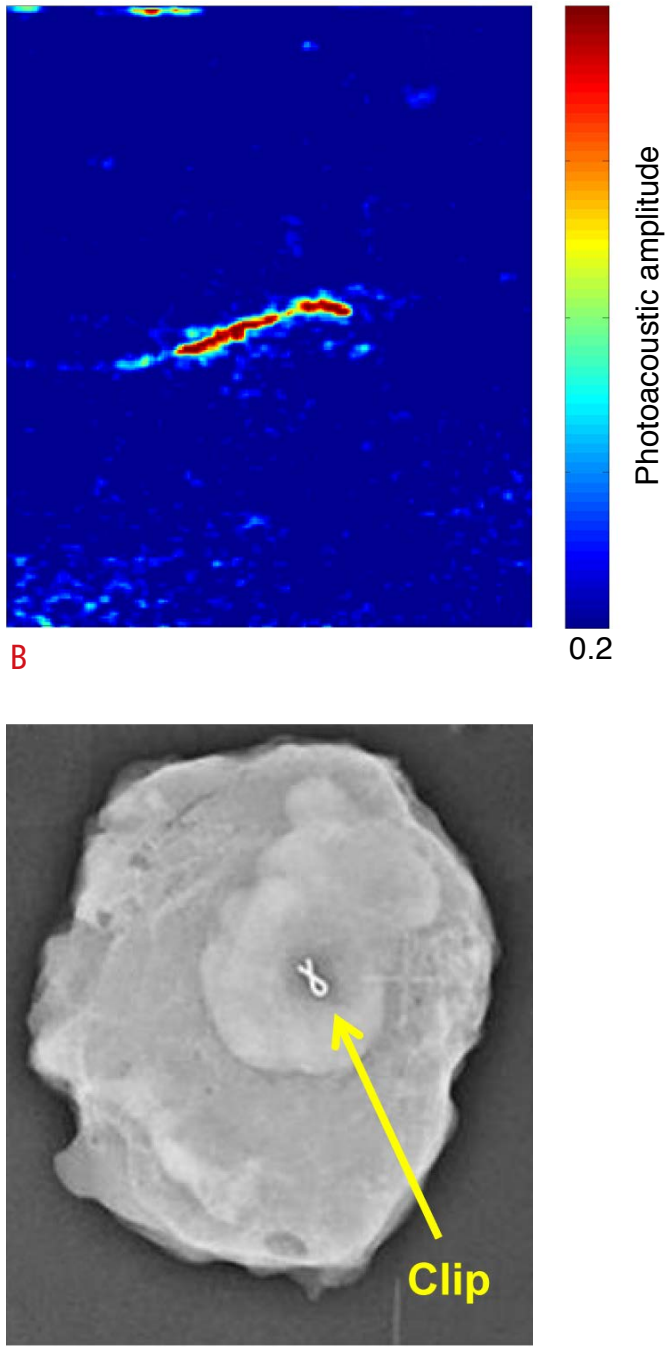

D

Fig. 5. Identification of sentinel lymph node (SLN) in breast cancer patients using photoacoustic imaging.

A. Sonogram shows lymph node in a breast cancer patient. B. Photoacoustic image shows a strong signal from the lymph node due to methylene blue accumulation confirming the ultrasound detected node as a SLN. C. Co-registered photoacoustic image of the SLN overlaid on sonogram. D. Radiograph of surgically removed SLN shows the presence of tissue-marking titanium clip which was implanted under the guidance of photoacoustic imaging, validating the feasibility of photoacoustic imaging to identify SLNs noninvasively. Reproduced from Garcia-Uribe A et al. Sci Rep 2015;5:15748 [75], according to the Creative Commons license. 


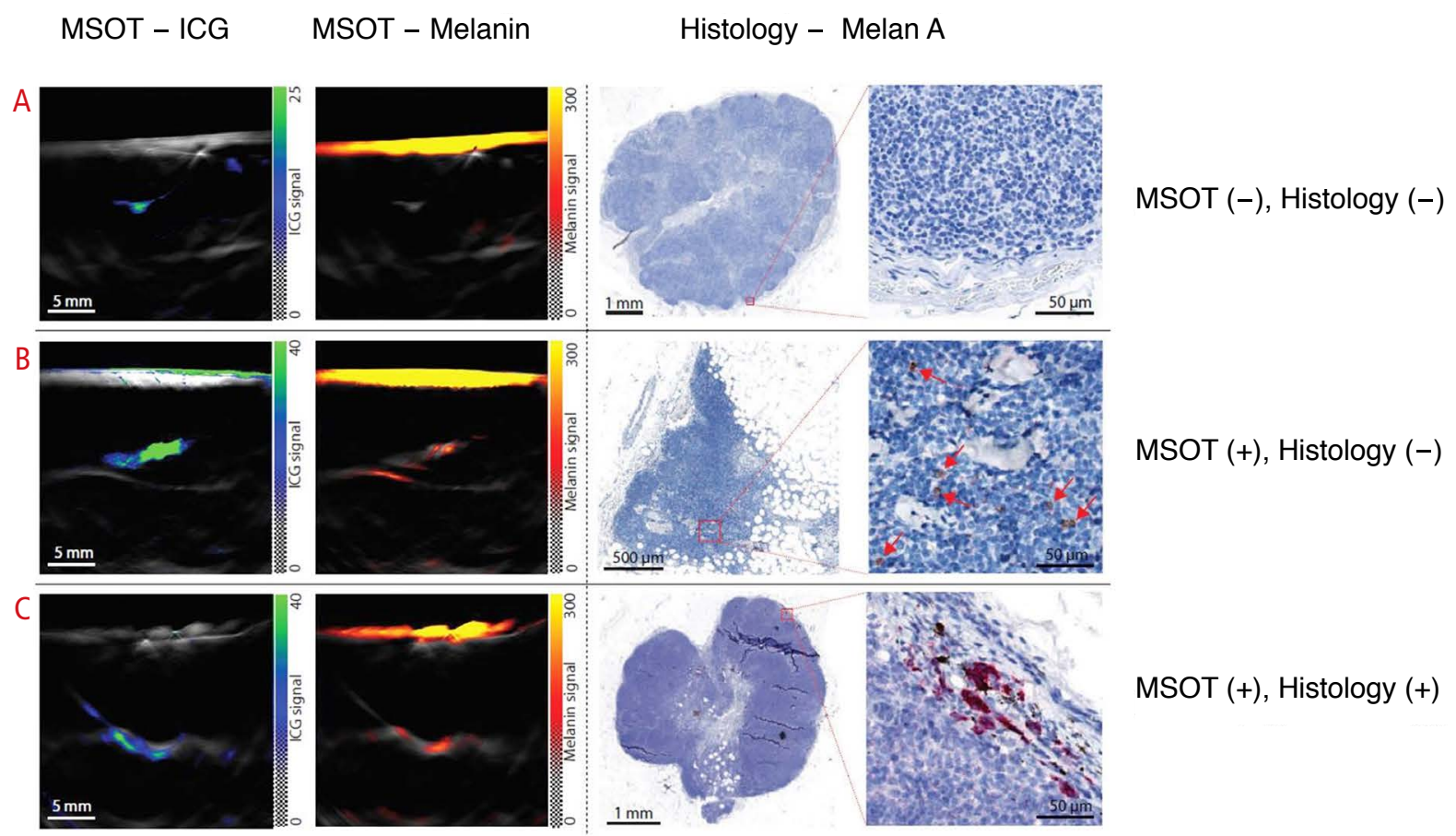

Fig. 6. Sentinel lymph node (SLN) visualization using a handheld multispectral optoacoustic tomography (MSOT) transducer in patients with melanoma.

Indocyanine green (ICG) dye was injected peritumorally to visualize the lymphatic drainage using photoacoustic imaging. While ICG indicates the location of SLNs, melanin inside the SLN suggests melanoma metastasis. A. Absence of melanin signal in ICG-positive right axillary node suggests that the patient has no detectable metastasis, confirmed as such by histology. B. Photoacoustic imaging of left axillary node (ICGpositive) in a patient suggests metastasis as indicated by strong melanin signal. Immunohistochemistry revealed no evidence of metastasis when stained for Melan A indicating false-positive photoacoustic imaging diagnosis. Left axillary lymph node in a patient showing high melanin signal on photoacoustic image (C) which was confirmed to be metastatic on histology (true positive finding). Adapted from Stoffels I et al. Sci Transl Med 2015;7:317ra199 [71], with permission of The American Association for the Advancement of Science.

false-negatives reported, a sensitivity of $100 \%$ and specificity of $48.6 \%$ were reported.

\section{Challenges and Future Outlook}

From this review, one can appreciate the complexity of categorizing photoacoustic imaging systems which are evolving into more robust equipment to facilitate clinical adoption. It can also be noted that the majority of the systems discussed here have been designed primarily at academic institutions, with only a few exceptions. These systems are therefore one-of-a-kind prototypes rather than final products. Since these systems were not designed for manufacturability and were developed to demonstrate feasibility with reasonable safety, they suffer from limited standardization across the spectrum making it almost impossible to compare data between the systems posing a didactic challenge for the clinicians, not to mention the regulatory hurdles. Developments in system design to assume the form factor of clinical ultrasound scanners as suggested by recent advances is a step in the right direction to facilitate faster clinical integration filling this void. For photoacoustic imaging systems to be adopted widely, fast lasers [80] that can operate at higher frame rates (hundreds or thousands of Hertz) similar to ultrasound for real-time image acquisition are important along with adequate safety monitoring systems that ensure safe laser-exposure levels to patient and the provider [81].

Another key factor which impacts the clinical adoption is the approval for marketing of the device for a specific clinical indication by regional regulatory bodies such as FDA in the United States and CE Mark in European Union. In light of Affordable Care Act adopted in United States recently which imposes reimbursement penalties to healthcare facilities based on quality metrics, it has become increasingly complex for the medical device companies to bring new technologies for patient care as the healthcare sector is undergoing a major shift from fee-for-service model to valuebased model requiring companies to demonstrate value proposition clearly over existing standard-of-care at similar or lower adoption 
costs. Therefore, it is important that the inventors consider such macroeconomic factors while developing commercial photoacoustic imaging systems in order to ensure a successful clinical translation. Several clinical trials on photoacoustic imaging [82-89] which are currently underway (mostly first-in-human or phase I) focus on critical endpoints to demonstrate feasibility, safety and effectiveness. These trials, if successful, enable the need for subsequent larger phase II clinical trials that need to demonstrate value proposition to establish a robust reimbursement pathway which is a crucial factor for successful clinical adoption in addition to the safety and effectiveness data typically acquired for regulatory purposes. Formal health technology assessment programs which are more common in Europe than in United States $[90,91]$ can be helpful during the early stages of photoacoustic imaging device development to anticipate any costs, challenges and pitfalls upfront and help mitigate the risks associated with business development and clinical adoption.

\section{Conclusion}

There is a growing interest in photoacoustic imaging within the key stakeholders including clinicians with several ongoing first-inhuman clinical trials, as well as large medical device companies that are cautiously eager to acquire new technologies which are effective, and carry minimal regulatory and reimbursement risks allowing market penetration and adoption. Clinical applications discussed here highlight the potential of clinical photoacoustic imaging to complement ultrasound and other existing armaments to help combat cancer and hopefully ultimately ease its burden on the global healthcare system.

ORCID: Keerthi S. Valluru: http://orcid.org/0000-0002-8627-973X; Juergen K. Willmann: http://orcid.org/0000-0002-9517-4657

\section{Conflict of Interest}

No potential conflict of interest relevant to this article was reported.

\section{Acknowledgments}

This work was supported by NIH R21EB022214 (JKW) and R01CA155289 (JKW). We thank James Strommer for his assistance with Fig. 1.

\section{References}

1. Willmann JK, van Bruggen N, Dinkelborg LM, Gambhir SS. Molecular imaging in drug development. Nat Rev Drug Discov 2008; 7:591-607.

2. Pysz MA, Willmann JK. Targeted contrast-enhanced ultrasound: an emerging technology in abdominal and pelvic imaging.
Gastroenterology 2011;140:785-790.

3. Kircher MF, Hricak H, Larson SM. Molecular imaging for personalized cancer care. Mol Oncol 2012;6:182-195.

4. Hussain T, Nguyen QT. Molecular imaging for cancer diagnosis and surgery. Adv Drug Deliv Rev 2014;66:90-100.

5. Seaman ME, Contino G, Bardeesy N, Kelly KA. Molecular imaging agents: impact on diagnosis and therapeutics in oncology. Expert Rev Mol Med 2010;12:e20.

6. Sippel S, Muruganandan K, Levine A, Shah S. Review article: use of ultrasound in the developing world. Int J Emerg Med 2011;4:72.

7. Abou-Elkacem L, Bachawal SV, Willmann JK. Ultrasound molecular imaging: moving toward clinical translation. Eur J Radiol 2015;84:1685-1693.

8. Bachawal SV, Jensen KC, Wilson KE, Tian L, Lutz AM, Willmann JK. Breast cancer detection by $\mathrm{B} 7-\mathrm{H} 3$-targeted ultrasound molecular imaging. Cancer Res 2015;75:2501-2509.

9. Kircher MF, Willmann JK. Molecular body imaging: MR imaging, CT, and US. part I. principles. Radiology 2012;263:633-643.

10. Kircher MF, Willmann JK. Molecular body imaging: MR imaging, CT, and US. Part II. Applications. Radiology 2012;264:349-368.

11. Jeong WK, Lim HK, Lee HK, Jo JM, Kim Y. Principles and clinical application of ultrasound elastography for diffuse liver disease. Ultrasonography 2014;33:149-160.

12. Lee SH, Chang JM, Cho N, Koo HR, Yi A, Kim SJ, et al. Practice guideline for the performance of breast ultrasound elastography. Ultrasonography 2014;33:3-10.

13. Kwak JY, Kim EK. Ultrasound elastography for thyroid nodules: recent advances. Ultrasonography 2014;33:75-82.

14. Ginat DT, Destounis SV, Barr RG, Castaneda B, Strang JG, Rubens DJ. US elastography of breast and prostate lesions. Radiographics 2009;29:2007-2016.

15. Cho N, Jang M, Lyou CY, Park JS, Choi HY, Moon WK. Distinguishing benign from malignant masses at breast US: combined US elastography and color doppler US: influence on radiologist accuracy. Radiology 2012;262:80-90.

16. Valluru KS, Wilson KE, Willmann JK. Photoacoustic Imaging in oncology: translational preclinical and early clinical experience. Radiology 2016;280:332-349.

17. Xu M, Wang LV. Photoacoustic imaging in biomedicine. Rev Sci Instrum 2006;77:041101.

18. Hanahan D, Weinberg RA. Hallmarks of cancer: the next generation. Cell 2011;144:646-674.

19. Wilson KE, Bachawal SV, Tian L, Willmann JK. Multiparametric spectroscopic photoacoustic imaging of breast cancer development in a transgenic mouse model. Theranostics 2014;4:1062-1071.

20. Bernsen MR, Kooiman K, Segbers M, van Leeuwen FW, de Jong M. Biomarkers in preclinical cancer imaging. Eur J Nucl Med Mol Imaging 2015;42:579-596.

21. Bell AG. Production of sound by radiant energy. J Franklin Inst 1881;111:401-426. 
22. Valluru KS, Chinni BK, Rao NA, Bhatt S, Dogra VS. Basics and clinical applications of photoacoustic imaging. Ultrasound Clin 2009;4:403-429.

23. Guo Z, Li L, Wang LV. On the speckle-free nature of photoacoustic tomography. Med Phys 2009;36:4084-4088.

24. Vogel A, Venugopalan V. Mechanisms of pulsed laser ablation of biological tissues. Chem Rev 2003;103:577-644.

25. Kim J, Lee D, Jung U, Kim C. Photoacoustic imaging platforms for multimodal imaging. Ultrasonography 2015;34:88-97.

26. Xia J, Yao J, Wang LV. Photoacoustic tomography: principles and advances. Electromagn Waves (Camb) 2014;147:1-22.

27. Weissleder R. A clearer vision for in vivo imaging. Nat Biotechnol 2001;19:316-317.

28. Frangioni JV. In vivo near-infrared fluorescence imaging. Curr Opin Chem Biol 2003;7:626-634.

29. Jacques SL. Optical properties of biological tissues: a review. Phys Med Biol 2013;58:R37-R61.

30. Friebel M, Roggan A, Muller G, Meinke M. Determination of optical properties of human blood in the spectral range 250 to $1100 \mathrm{~nm}$ using Monte Carlo simulations with hematocrit-dependent effective scattering phase functions. J Biomed Opt 2006;11:34021.

31. Sethuraman S, Amirian JH, Litovsky SH, Smalling RW, Emelianov SY. Spectroscopic intravascular photoacoustic imaging to differentiate atherosclerotic plaques. Opt Express 2008;16:3362-3367.

32. Beard P. Biomedical photoacoustic imaging. Interface Focus 2011;1:602-631.

33. Allen TJ, Hall A, Dhillon AP, Owen JS, Beard PC. Spectroscopic photoacoustic imaging of lipid-rich plaques in the human aorta in the 740 to $1400 \mathrm{~nm}$ wavelength range. J Biomed Opt 2012;17:061209.

34. Wang X, Xie X, Ku G, Wang LV, Stoica G. Noninvasive imaging of hemoglobin concentration and oxygenation in the rat brain using high-resolution photoacoustic tomography. J Biomed Opt 2006;11:024015.

35. Kim JW, Dang CV. Cancer's molecular sweet tooth and the Warburg effect. Cancer Res 2006;66:8927-8930.

36. Cairns RA, Harris IS, Mak TW. Regulation of cancer cell metabolism. Nat Rev Cancer 2011;11:85-95.

37. Razansky D, Baeten J, Ntziachristos V. Sensitivity of molecular target detection by multispectral optoacoustic tomography (MSOT). Med Phys 2009;36:939-945.

38. Hannah A, Luke G, Wilson K, Homan K, Emelianov S. Indocyanine green-loaded photoacoustic nanodroplets: dual contrast nanoconstructs for enhanced photoacoustic and ultrasound imaging. ACS Nano 2014;8:250-259.

39. Kim G, Huang SW, Day KC, O'Donnell M, Agayan RR, Day MA, et al. Indocyanine-green-embedded PEBBLEs as a contrast agent for photoacoustic imaging. J Biomed Opt 2007;12:044020.

40. Ashkenazi S. Photoacoustic lifetime imaging of dissolved oxygen using methylene blue. J Biomed Opt 2010;15:040501.

41. Song KH, Stein EW, Margenthaler JA, Wang LV. Noninvasive photoacoustic identification of sentinel lymph nodes containing methylene blue in vivo in a rat model. J Biomed Opt 2008;13:054033.

42. Luke GP, Yeager D, Emelianov SY. Biomedical applications of photoacoustic imaging with exogenous contrast agents. Ann Biomed Eng 2012;40:422-437.

43. Conde J, Doria G, Baptista P. Noble metal nanoparticles applications in cancer. J Drug Deliv 2012;2012:751075.

44. De la Zerda A, Zavaleta C, Keren S, Vaithilingam S, Bodapati S, Liu $Z$, et al. Carbon nanotubes as photoacoustic molecular imaging agents in living mice. Nat Nanotechnol 2008;3:557-562.

45. Wilson K, Homan K, Emelianov S. Biomedical photoacoustics beyond thermal expansion using triggered nanodroplet vaporization for contrast-enhanced imaging. Nat Commun 2012;3:618.

46. Strohm EM, Gorelikov I, Matsuura N, Kolios MC. Acoustic and photoacoustic characterization of micron-sized perfluorocarbon emulsions. J Biomed Opt 2012;17:096016.

47. Weber J, Beard PC, Bohndiek SE. Contrast agents for molecular photoacoustic imaging. Nat Methods 2016;13:639-650.

48. Zackrisson S, van de Ven SM, Gambhir SS. Light in and sound out: emerging translational strategies for photoacoustic imaging. Cancer Res 2014;74:979-1004.

49. Yang X, Stein EW, Ashkenazi S, Wang LV. Nanoparticles for photoacoustic imaging. Wiley Interdiscip Rev Nanomed Nanobiotechnol 2009;1:360-368.

50. Wilson KE, Wang TY, Willmann JK. Acoustic and photoacoustic molecular imaging of cancer. J Nucl Med 2013;54:1851-1854.

51. Wang LV. Tutorial on photoacoustic microscopy and computed tomography. IEEE J Sel Top Quantum Electron 2008;14:171-179.

52. Mehrmohammadi M, Yoon SJ, Yeager D, Emelianov SY. Photoacoustic imaging for cancer detection and staging. Curr Mol Imaging 2013;2:89-105.

53. Oeffinger KC, Fontham ET, Etzioni R, Herzig A, Michaelson JS, Shih $Y C$, et al. Breast cancer screening for women at average risk: 2015 guideline update from the American Cancer Society. JAMA 2015;314:1599-1614.

54. Piras D, Xia W, Steenbergen W, Van Leeuwen TG, Manohar S. Photoacoustic imaging of the breast using the twente photoacoustic mammoscope: present status and future perspectives. IEEE J Sel Top Quantum Electron 2010;16:730-739.

55. Heijblom $M$, Piras $D$, van den Engh FM, van der Schaaf $M$, Klaase JM, Steenbergen $W$, et al. The state of the art in breast imaging using the Twente Photoacoustic Mammoscope: results from 31 measurements on malignancies. Eur Radiol 2016 Mar 5 [Epub]. http://dx.doi.org/10.1007/s00330-016-4240-7.

56. Hilgerink MP, Hummel MJ, Manohar S, Vaartjes SR, ljzerman MJ. Assessment of the added value of the Twente Photoacoustic Mammoscope in breast cancer diagnosis. Med Devices (Auckl) 
2011:4:107-115.

57. Kitai T, Torii M, Sugie $T$, Kanao S, Mikami $Y$, Shiina $T$, et al. Photoacoustic mammography: initial clinical results. Breast Cancer 2014;21:146-153.

58. Fakhrejahani E, Torii M, Kitai T, Kanao S, Asao Y, Hashizume Y, et al. Clinical report on the first prototype of a photoacoustic tomography system with dual illumination for breast cancer imaging. PLoS One 2015;10:e0139113.

59. Kim GR, Kang J, Kwak JY, Chang JH, Kim SI, Youk JH, et al. Photoacoustic imaging of breast microcalcifications: a preliminary study with 8-gauge core-biopsied breast specimens. PLoS One 2014;9:e105878.

60. Favazza CP, Jassim O, Cornelius LA, Wang LV. In vivo photoacoustic microscopy of human cutaneous microvasculature and a nevus. J Biomed Opt 2011;16:016015.

61. Dogra VS, Chinni BK, Valluru KS, Joseph JV, Ghazi A, Yao JL, et al. Multispectral photoacoustic imaging of prostate cancer: preliminary ex-vivo results. J Clin Imaging Sci 2013;3:41.

62. Valluru KS, Chinni BK, Rao NA, Bhatt S, Dogra VS. Development of a c-scan photoacoutsic imaging probe for prostate cancer detection. In: SPIE Proceedings Vol. 7968. Medical Imaging 2011: Ultrasonic Imaging, Tomography, and Therapy; 2011 Feb 12; Lake Buena Vista, FL, USA. Bellingham, WA: Society of Photo-Optical Instrumentation Engineers, 2011;79680C.

63. Dogra VS, Chinni BK, Valluru KS, Moalem J, Giampoli EJ, Evans K, et al. Preliminary results of ex vivo multispectral photoacoustic imaging in the management of thyroid cancer. AJR Am J Roentgenol 2014;202:W552-W558.

64. Aguirre A, Ardeshirpour Y, Sanders MM, Brewer M, Zhu Q. Potential role of coregistered photoacoustic and ultrasound imaging in ovarian cancer detection and characterization. Transl Oncol 2011;4:29-37.

65. Kumavor PD, Alqasemi U, Tavakoli B, Li H, Yang Y, Sun X, et al. Coregistered pulse-echo/photoacoustic transvaginal probe for real time imaging of ovarian tissue. J Biophotonics 2013;6:475-484.

66. Alqasemi U, Li H, Yuan G, Aguirre A, Zhu Q. Ultrafast ultrasound and photoacoustic co-registered imaging system based on FPGA parallel processing. In: SPIE Proceedings Vol. 8223. Photons Plus Ultrasound: Imaging and Sensing 2012; 2012 Jan 21; San Francisco, CA, USA. Bellingham, WA: Society of Photo-Optical Instrumentation Engineers, 2012;82232U.

67. Peng K, He L, Wang B, Xiao J. Detection of cervical cancer based on photoacoustic imaging-the in-vitro results. Biomed Opt Express 2015;6:135-143.

68. Ermilov SA, Khamapirad T, Conjusteau A, Leonard MH, Lacewell R, Mehta $\mathrm{K}$, et al. Laser optoacoustic imaging system for detection of breast cancer. J Biomed Opt 2009;14:024007.

69. Kruger RA, Kuzmiak CM, Lam RB, Reinecke DR, Del Rio SP, Steed D. Dedicated 3D photoacoustic breast imaging. Med Phys
2013;40:113301.

70. Jose J, Grootendorst DJ, Vijn TW, Wouters MW, van Boven H, van Leeuwen TG, et al. Initial results of imaging melanoma metastasis in resected human lymph nodes using photoacoustic computed tomography. J Biomed Opt 2011;16:096021.

71. Stoffels I, Morscher S, Helfrich I, Hillen U, Leyh J, Burton NC, et al. Metastatic status of sentinel lymph nodes in melanoma determined noninvasively with multispectral optoacoustic imaging. Sci Transl Med 2015;7:317ra199.

72. Ermilov SA, Fronheiser MP, Brecht HP, Su R, Conjusteau A, Mehta $\mathrm{K}$, et al. Development of laser optoacoustic and ultrasonic imaging system for breast cancer utilizing handheld array probes. In: SPIE Proceedings Vol. 7177. Photons Plus Ultrasound: Imaging and Sensing 2009; 2009 Jan 24; San Jose, CA, USA. Bellingham, WA: Society of Photo-Optical Instrumentation Engineers, 2009;717703.

73. Stavros AT, Lavin P, Schoenfeld D, Ulissey MJ. Breast mass classification based on opto-acoustic features using data mining. In: 2014 ECR Annual Meeting; 2014 Mar 6-10; Vienna, Austria; C-0926.

74. Stavros AT, Lerner A, Tucker L, Boyd B, Fine R, Burak W. Optoacoustic imaging in the evaluation of BI-RADS 3 lesions: findings and implications. In: National Consortium of Breast Centers 2014; 2014 Mar 15-19; Las Vegas, NV, USA.

75. Garcia-Uribe A, Erpelding TN, Krumholz A, Ke H, Maslov K, Appleton $C$, et al. Dual-modality photoacoustic and ultrasound imaging system for noninvasive sentinel lymph node detection in patients with breast cancer. Sci Rep 2015;5:15748.

76. Erpelding TN, Garcia-Uribe A, Krumholz A, Ke H, Maslov K, Appleton $C$, et al. A dual-modality photoacoustic and ultrasound imaging system for noninvasive sentinel lymph node detection: preliminary clinical results. In: SPIE Proceedings Vol. 8943. Photons Plus Ultrasound Imaging and Sensing 2014; 2014 Feb 1; San Francisco, CA, USA. Bellingham, WA: Society of Photo-Optical Instrumentation Engineers, 2014;894359.

77. Neuschmelting $V$, Burton NC, Lockau $H$, Urich $A$, Harmsen $S$,

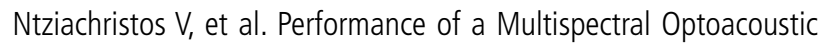
Tomography (MSOT) System equipped with 2D vs. 3D handheld probes for potential clinical translation. Photoacoustics 2016;4:110.

78. Buehler A, Kacprowicz M, Taruttis A, Ntziachristos V. Realtime handheld multispectral optoacoustic imaging. Opt Lett 2013:38:1404-1406.

79. Taruttis $A$, Timmermans $A C$, Wouters $P C$, Kacprowicz M, van Dam GM, Ntziachristos V. Optoacoustic imaging of human vasculature: feasibility by using a handheld probe. Radiology 2016:152160.

80. Sivasubramanian K, Pramanik M. High frame rate photoacoustic imaging at 7000 frames per second using clinical ultrasound system. Biomed Opt Express 2016;7:312-323.

81. Laser Institute of America. American National Standard for Safe Use 
of Lasers. New York: Laser Institute of America, 2007.

82. M.D. Anderson Cancer Center. LOUIS-3D Breast Study [Internet]. Bethesda, MD: U.S. National Library of Medicine, 2012 [cited 2015 Feb 22]. Available from: https://clinicaltrials.gov/ct2/show/ NCT01755130.

83. Seno Medical Instruments Inc. The PIONEER-01 Pivotal Study of the Imagio Breast Imaging System [Internet]. Bethesda, MD: U.S. National Library of Medicine, 2013 [cited 2015 Feb 22]. Available from: https://clinicaltrials.gov/ct2/show/NCT01943916.

84. Carson PL. Simulated Screening Study for Breast Imaging [Internet]. Bethesda, MD: U.S. National Library of Medicine, 2012 [cited 2015 Feb 22]. Available from: https://clinicaltrials.gov/ct2/show/ NCT01807754.

85. Heijblom M. Breast imaging using light and sound [Internet]. Amsterdam: Nederlands Trial Register, 2011 [cited 2015 Feb 22]. Available from: http://www.trialregister.nl/trialreg/admin/rctview. asp?TC=2945.

86. Photoacoustic tomography (PAT) in combination with high resolution ultrasound for non-invasive diagnosis of sentinel lymph node in patients with malignant melanoma [Internet]. Freiburg:
German Clinical Trials Register, 2013 [cited 2015 Feb 22]. Available from: https://drks-neu.uniklinik-freiburg.de/drks_web/navigate. do? navigationld=trial.HTML\&TRIAL_ID=DRKS00005447.

87. University of Arkansas. In vivo real-time detection of circulating melanoma cells [Internet]. Bethesda, MD: U.S. National Library of Medicine, 2013 [cited 2015 Feb 22]. Available from: https:// clinicaltrials.gov/ct2/show/NCT01776905.

88. Gambhir SS. Photoacoustic imaging (PAI) of the prostate: a clinical feasibility study [Internet]. Bethesda, MD: U.S. National Library of Medicine, 2012 [cited 2015 Feb 22]. Available from: https:// clinicaltrials.gov/ct2/show/NCT01551576.

89. Nagengast WB. Visualization of rectal cancer during endoscopy, using a fluorescent tracer (RAPIDO-TRACT) [Internet]. Bethesda, MD: U.S. National Library of Medicine, 2012 [cited 2015 Feb 22]. Available from: https://clinicaltrials.gov/ct2/show/NCT01972373.

90. Sullivan SD, Watkins J, Sweet B, Ramsey SD. Health technology assessment in health-care decisions in the United States. Value Health 2009;12 Suppl 2:S39-S44.

91. Luce $B$, Cohen RS. Health technology assessment in the United States. Int J Technol Assess Health Care 2009;25 Suppl 1:33-41. 